\title{
Crossing Number of Abstract Topological Graphs
}

\author{
Jan Kratochvíl` \\ Charles University, Prague, Czech Republic \\ honza@kam.ms.mff.cuni.cz
}

\begin{abstract}
We revoke the problem of drawing graphs in the plane so that only certain specified pairs of edges are allowed to cross. We overview some previous results and open problems, namely the connection to intersection graphs of curves in the plane. We complement these by stating a new conjecture and showing that its proof would solve the problem of algorithmic decidability of recognition of string graphs as well as realizability of abstract topological graphs and feasible drawability of graphs with restricted edge crossings.
\end{abstract}

\section{Drawing Graphs When Only Some Pairs of Egdes Are Allowed to Cross}

Minimizing the number of crossing points in planar drawings of graphs in the plane is an important task. Too many crossing points - too dense a drawing makes the chart badly readable for human eyes. It is well known that finding the exact minimum number of crossing points needed for planar drawing of a given graph, the so called crossing number, is an NP-hard problem [1]. On the other hand, this problem was deeply studied and well understood for many special graph classes and many exact results or at least tight bounds are known [6]. Much less studied is the question of drawing graphs for which only some pairs of egdes are allowed to cross. This natural generalization of planar graphs on one hand and crossing numbers on the other one was introduced in [4]. Practical motivation for this question are e.g. VLSI layouts with further restrictions - edges of certain types may not be allowed to cross because of their actual realization by connectors made from certain materials with different properties. Graph theoretical and complexity consequences of the concept of 'allowing only certain pairs of edges to cross' may bee even more interesting. Among them is the astonishing and challenging fact that so far there is no recursive algorithm known to decide the existence of a feasible drawing in this sense.

In this note we want to reattract attention to the question of allowed crossings. We briefly review the definitions and give an overview of known results

\footnotetext{
* The author acknowledges partial support from Czech Research Grants GAUK 193 and 194 and GAČR 201/1996/0194, and Czech-US Science and Technology Research grant No. 94051. Mailing address: KAM MFF UK, Malostranské nám. 25, 11800 Praha 1, Czech Republic.
} 
and open problems. In the last section we report on a moderate progress in the question of recognition. Namely we introduce a new conjecture which, if settled in affirmative, would imply an exponential upper bound on the crossing number, and hence the existence of an (exponential but) finite recognition algorithm.

A topological graph is a graph drawn in the plane with any number of edge crossings. However, some basic constraints are assumed, such as drawings of edges do not pass through vertices, any two edges share only a finite number of common points and they cross (i.e., do not touch) in the neighborhood of every common inner point (thus called a crossing point). No three edges pass through the same crossing point. A topological graph is thus a pair $(G, D)$ where $G$ is an abstract graph (i.e., $G=(V, E)$ where $V$ is a finite set of vertices and $E \subset\left(\begin{array}{c}V \\ 2\end{array}\right)$ is a set of edges, we consider undirected graphs without loops or multiple edges) and $D$ is the drawing of $G$ ( $D$ is a mapping from $V \cup E$ into points and Jordan arcs in the plane such that $D(v)$ is the point representing vertex $v$ in the drawing $D$ and $D(e)$ is the arc representing edge $e$, note that the arcs representing edges are considered open, i.e., their endpoints do not belong to the arcs). The drawing determines the set of crossing pairs of edges $R_{D}=\{\{e, f\}: D(e) \cap D(f) \neq \emptyset\}$.

The first natural question is to reverse the reasoning. A pair $(G, R)$ is called an abstract topological graph (briefly an AT-graph) if $G$ is a graph and $R \subset\left(\begin{array}{c}E \\ 2\end{array}\right)$ is a set of pairs of its edges. (We use this notion as only crossing pairs of edges are specified, but not the actual drawing.) Then it is natural to ask if $G$ allows a drawing $D$ such that $R_{D}=R$. Such a drawing (if it exists) is called a realization of $(G, R)$. It is proved in [3] that it is NP-hard to decide if a given AT-graph is realizable in this sense. Though this approach has applications (e.g. for string graphs, see the next section), from the Graph Drawing point of view it seems more natural to ask whether $(G, R)$ has a drawing $D$ such that $R_{D} \subseteq R$, i.e., whether $G$ can be drawn in the plane so that only pairs of edges listed in $R$ are allowed to cross. In [4, the AT-graph is called weak realizable if such a drawing exists. Let us call such a drawing feasible in this note. Obviously not every AT-graph has a feasible drawing, e.g., if $R=\emptyset$ then $(G, \emptyset)$ has a feasible drawing if and only if $G$ is planar. However, unlike planar graphs, it is NP-hard to decide if a given AT-graph allows a feasible drawing [3]. But the situation is even more serious than NP-completeness. So far no finite algorithm is known to decide realizability and weak realizability of AT-graphs, and it is even known that straightforward strategy to place the decision problem in the class NP fails (see the next section). In some sense this makes the problem theoretically more interesting, since there are not many graph theoretical problems with practical motivation which float above the class NP. (Perhaps it is the topological nature of the problem that causes this effect. Another problem for which no finite algorithm was known for a while, are linklessly embedabble graphs, now polynomial by Robertson-Seymour Graph Minor Machinery.) 

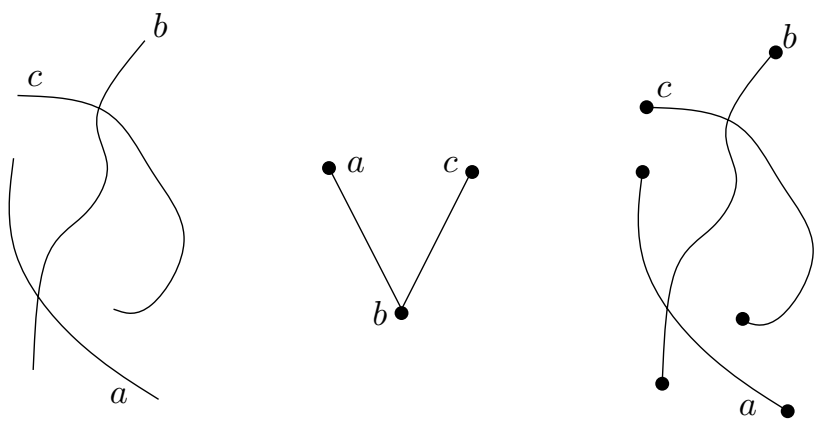

$G$

$$
R=\left\{\begin{array}{c}
3 K_{2} \\
R a, b\},\{b, c\}\}
\end{array}\right.
$$

Fig. 1. String graphs as representations of AT-graphs.

\section{Abstract Topological Graphs and Intersection Graphs}

Intersection representations of graphs (namely by planar objects) have many applications and there is now an extensive literature devoted to interval graphs, circle graphs, permutation graphs, cocomparability graphs and other intersection defined classes of graphs (cf. e.g. 2]). String graphs (intersection graphs of curves in the plane) form the most general of them (they are exactly the intersection graphs of arc-connected sets in the plane). String graphs are fully expressible in the language of AT-graphs: Given a graph $G=(V, E)$ on $n$ vertices, consider the matching $n K_{2}$ and give its edges the names of the vertices of $G$. Then $G$ a is an intersection graph of curves if and only if the AT-graph $\left(n K_{2}, E\right)$ is realizable. In fact, it is shown in [3] that recognizing string graphs is NP-hard, via a reduction from realizability of AT-graphs. Similarly as in the case of realizability of ATgraphs, no finite algorithm for recognition of string graphs is known.

\section{Crossing Number of AT-Graphs}

Definition 31 The crossing number (denoted by $\mathrm{cr}_{a t}(G, R)$ ) of an AT-graph $(G, R)$ is the minimum possible number of crossing points in a feasible drawing of $(G, R)$. We denote by $\mathrm{cr}_{a t}(m)$ the maximum of $c r_{a t}(G, R)$ taken over the class of all feasibly drawable AT-graphs $(G, R)$ with $G$ having at most $m$ edges.

As a special case, $c r_{a t}(G, R)=\operatorname{cr}(G)$ if $R=\left(\begin{array}{c}E(G) \\ 2\end{array}\right)$ allows any pair of edges to cross (here $\operatorname{cr}(G)$ denotes the traditional unrestricted crossing number of a graph $G$, where the crossing number is the minimum total number of crossing points in a planar drawing). Note that $c r_{a t}(m)$ is finite for every $m$, since the maximum is taken over a finite set of AT-graphs. No upper bound, is however, known:

Problem 1 Is there a recursive upper bound for $\mathrm{cr}_{a t}(m)$ ? 

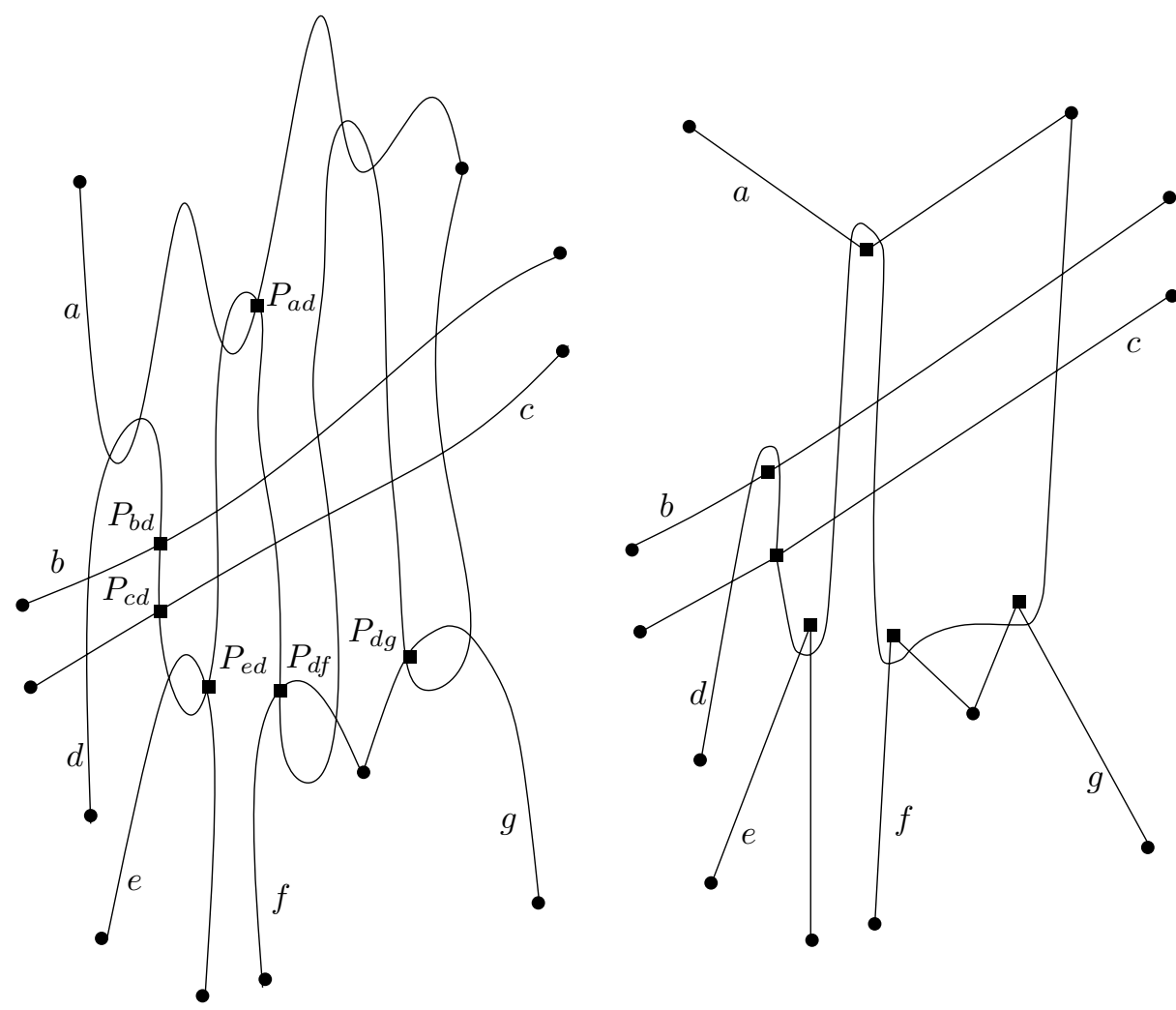

Fig. 2. Reducing the number of crossing points in an AT-graph realization.

Note that proving any recursive upper bound would imply the existence of a finite recognition algorithm. One would just try all possible placements of crossing points on the edges of $G$ and test planarity.

The same argument applies to AT-graph realizability as well: Given a realizable AT-graph $(G, R)$ with $m$ edges, fix a realization and for any pair $\{e, f\} \in R$, choose one crossing point $P_{\text {ef }}$ of the drawings of the edges $e$ and $f$. Regard the sections of the edges of $G$ between the marked crossing points (and between marked crossing points and vertices of $G$ ) as edges of a new graph $G^{\prime}$ (with vertex set $\left.V(G) \cup\left\{P_{e f} \mid\{e, f\} \in R\right\}\right)$. Call this drawing $D^{\prime}$. Now $G^{\prime}$ has at most $m^{2}$ edges (every edge of $G$ has at most $m-1$ marked crossing points with other edges), and hence $c r_{a t}\left(G^{\prime}, R^{\prime}=R_{D^{\prime}}\right) \leq c r_{a t}\left(m^{2}\right)$. Every feasible drawing of $\left(G^{\prime}, R^{\prime}\right)$ then determines a drawing of $G$ in which edges $e, f$ share the point $P_{e f}$, whenever $\{e, f\} \in R$. Some of these points may have become touching points, so after overlaping the curves near such points (creating two crossing points from one touch point) we get a full realization of $(G, R)$ with at most $2 c r_{a t}\left(m^{2}\right)$ crossing points (cf. Figure 2 for illustration). Now the same argument as for feasible drawability applies - guess orderings of the crossing points along the edges and 
test planarity. Noting that string graphs are special types of AT-graphs, we have the following:

Observation 32 An affirmative answer to Problem 1 would guarantee the existence of finite algorithms for recognizing string graphs, realizability of AT-graphs and weak realizability of AT-graphs.

One may ask if AT-graphs can force large crossing numbers. Yes, they can. Here comes perhaps the first real difference between the crossing numbers of graphs and AT-graphs. While in every optimal (unrestricted) drawing of a graph every two edges share at most one crossing point (folklore), consider the example in Figure 3 The only pairs of edges that are allowed to cross are $\{a, b\},\{a, c\},\{a, d\},\{a, e\},\{b, f\},\{b, g\},\{b, h\}$. The frame $G-\{a, b\}$ has a unique planar embedding and thus the drawing in the figure is the only feasible drawing of the AT-graph (from topological point of view). The edges $a, b$ share at least 2 crossing points, and besides that, they also share a common vertex. This is another difference from ordinary crossing number - in every optimal (unrestricted) drawing of a graph no two edges incident with the same vertex cross (again folklore).

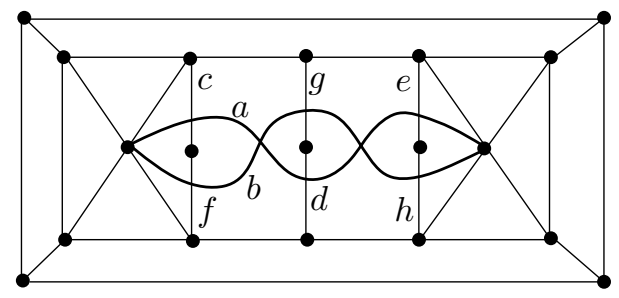

Fig. 3. AT-graph requiring double crossing.

It is somewhat surprising that there exist AT-graphs which require exponential number of crossing points:

Proposition 33 [5] For every large enough $m, c r_{a} t(m) \geq 2^{\frac{n}{6}}$.

One can read this result as follows: The first natural strategy to place the recognition problem of weakly realizable AT-graphs in the class NP, namely guessing the crossing points of the edges and checking planarity, necessarily fails, as there graphs which would require exponentially large underlying graph of the realization, and planarity checking would not be polynomial in the size of the actual input. (This of course does not prove that the recognition is not in NP.) It is conjectured in [5] that $c r_{a t}(m) \leq 2^{O\left(n^{c}\right)}$ for some contstant $c$. 


\section{Is There Always a Single Crossing?}

In this section we introduce a conjecture which, if true, would imply a single exponential upper bound for $c r_{a t}(m)$, thereby answering Problem 1 and proving the conjecture of $[5]$.

Conjecture 2 In any optimal feasible drawing of an AT-graph, any edge which is crossed by at least one other edge is crossed by some edge exactly once.

In a feasible drawing $D$ of $G$, let $c_{D}(e)$ denote the number of edges that cross $D(e)$.

Lemma 41 Assume Conjecture 2 is true. Let $D$ be an optimal feasible drawing of an AT-graph $(G, R)$. Then every edge e shares at most $2^{c_{D}(e)}-1$ crossing points with other edges.

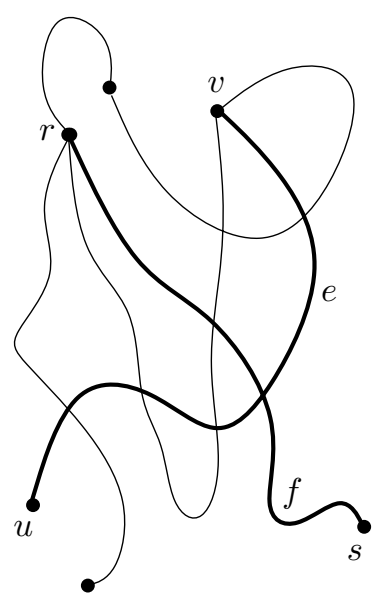

$D$

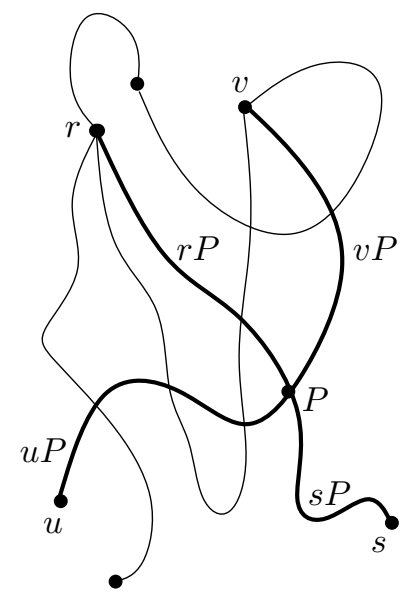

$D^{\prime}$

Fig. 4.

Proof: The proof goes by induction on the number of crossing points in $D$, i.e., on $\mathrm{cr}_{a t}(G, R)$. The statement is obviously true if $e$ is not crossed by other edges, i.e., $c_{D}(e)=0$. Suppose $c_{D}(e) \geq 1$. By Conjecture 2, there is an edge, say $f=r s$, which shares exactly one crossing point with $e$. Let $P$ be the crossing point of $D(e)$ and $D(f)$. Create a new graph $G^{\prime}$ with vertex set

$$
V^{\prime}=V(G) \cup\{P\}
$$

and edge set

$$
E^{\prime}=(E(G)-\{e, f\}) \cup\{u P, v P, r P, s P\} .
$$


We let $G^{\prime}$ resume the drawing $D$ of $G$, i.e., let $D^{\prime}$ be the drawing physically identical to $D$, such that $D^{\prime}(x)=D(x)$ for every vertex $x \in V(G), D^{\prime}(h)=D(h)$ for every edge $h \in E(G)-\{e, f\}, D^{\prime}(P)=P$ and the $\operatorname{arc} D(u) P$ on $D(e)$ is regarded as $D^{\prime}(u P)$, the arc $D(v) P$ on $D(e)$ is regarded as $D^{\prime}(v P)$ and similarly for $f=r s$. We define $R^{\prime}=R_{D^{\prime}}$ as the set of pairs of edges actually crossing in $D^{\prime}$. The drawing $D^{\prime}$ has one crossing less than $D$, i.e., $c r_{a t}(G, R)-1$. Hence $c r_{a t}\left(G^{\prime}, R^{\prime}\right) \leq c r_{a t}(G, R)-1$.

Suppose $\left(G^{\prime}, R^{\prime}\right)$ has a better feasible drawing, i.e., with less crossing points. Such a drawing would yield a feasible drawing of $(G, R)\left(\operatorname{setting} \tilde{D}(e)=D^{\prime}(u P) \cup\right.$ $\{P\} \cup D^{\prime}(v P)$ and $\tilde{D}(f)=D^{\prime}(r P) \cup\{P\} \cup D^{\prime}(s P)$, no restrictions of $R$ are violated and $\tilde{D}$ has at most one crossing point - $P$ - more than $\left.D^{\prime}\right)$. Since $D$ was an optimal feasible drawing of $(G, R)$, this is not possible. Hence $c r_{a t}\left(G^{\prime}, R^{\prime}\right)=$ $c r_{a t}(G, R)-1$.

It follows that $D^{\prime}$ is an optimal feasible drawing of $\left(G^{\prime}, R^{\prime}\right)$ with less crossing points than $D$, and we may apply induction hypothesis. Note that $c_{D^{\prime}}(u P) \leq$ $c_{D^{\prime}}(e)-1$ and $c_{D^{\prime}}(v P) \leq c_{D^{\prime}}(e)-1$ (since neither $f$ nor its descendants $r P, s P$ cross $e$ and in $D^{\prime}, e$ is allowed to cross only those edges that $e$ crossed in $D$ ). By induction hypothesis, $D^{\prime}(u P)$ shares at most

$$
2^{c_{D^{\prime}}(u P)}-1 \leq 2^{c_{D}(e)-1}-1
$$

crossing points with other edges, and $D^{\prime}(v P)$ shares at most

$$
2^{c_{D^{\prime}}(v P)}-1 \leq 2^{c_{D}(e)-1}-1
$$

crossing points with other edges. Going back to $D$, we see that $D(e)$ shares at most

$$
2^{c_{D^{\prime}}(u P)}-1+2^{c_{D^{\prime}}(v P)}-1+1 \leq 2 \cdot\left(2^{c_{D}(e)-1}-1\right)+1=2^{c_{D}(e)}-1
$$

crossing points with other edges.

Corollary 42 Suppose Conjecture 2 is true. Then $\mathrm{cr}_{a t}(m) \leq \frac{m}{2}\left(2^{m-1}-1\right)$.

Proof: Let $D$ be an optimal feasible drawing of an AT-graph $(G, R)$. Each of the $m$ edges is crossed by at most $m-1$ other edges, and hence each edge shares at most $2^{m-1}-1$ crossing points with other edges (by Lemma 41). This is in total $m\left(2^{m-1}-1\right)$, each being counted twice.

\section{Conclusion}

We believe that the question of minimizing the number of crossing points in feasible drawings of AT-graphs is interesting and worth further consideration. The main interest in author's eyes lies in Conjecture 2 which would settle the annoying fact that so far no finite algorithm for recognition of string graphs (nor of realizable AT-graphs) is known. Apart of this conjecture, determining bounds for crossing number of particular families of AT-graphs may be of separate interest. 


\section{References}

1. M.R.Garey, D.S.Johnson: Crossing number is NP-complete, SIAM J. Algebraic and Discrete Methods 4 (1983), 312-316

2. M.C.Golumbic: Algorithmic Graph theory and Perfect Graphs, Academic Press, New York, 1980

3. J.Kratochvíl: String graphs II. Recognizing string graphs is NP-hard, J. Combin. Theory Ser. B 52 (1991), 67-78

4. J.Kratochvíl, A. Lubiw, J. Nešetřil: Noncrossing subgraphs of topological layouts, SIAM J. Discrete Math. 4 (1991), 223-244

5. J.Kratochvíl, J. Matoušek: String graphs requiring exponential representations, J. Combin. Theory Ser. B 53 (1991), 1-4

6. F.Sharokhi, O.Sýkora, I.Vrto: Crossing numbers of graphs, lower bound techniques and algorithms: a survey, in: Proc. Graph Drawing '94, LNCS 894, Springer Verlag, Berlin, 1995, pp. 131-142 